\title{
Study on Performance of Anisotropic Materials of Thermal Conductivity
}

\author{
Shunyu Su*, Jian Chen and Chunzhi Zhang \\ College of Urban Construction, Wuhan University of Science and Technology, Wuhan 430070, China
}

\begin{abstract}
The advantage and shortcoming of anisotropic material of thermal conductivity using as building envelope were analyzed in this paper. The thermal performance of anisotropic material of thermal conductivity was presented by rotating the original rectangular axes so as to determine the principal axes and make the cross-derivative terms disappear. The results indicated that the heat flux vector is commonly not perpendicular to the isothermal surface in anisotropic material. Double skin facade has been successfully applied in many building designs. The application of laminated composite with anisotropic thermal conductivity in double skin envelope was proposed to avoid its disadvantage. The interior envelope in double skin system may be made of laminated glaze or other laminated materials. Basing on the combination of anisotropic material and double skin envelope, the indoor cooling and heating load decrease in summer and winter respectively while the anisotropic material was used as interior building envelope. Especially in summer, the effect of energy saving is obvious since the partial magnitude of conductive heat in the interior envelope could be brought out from the cavity by natural ventilation through it.
\end{abstract}

Keywords: Anisotropic material, double skin façade, laminated composite, thermal conductivity.

\section{INTRODUCTION}

Thermal conductivity of a material $k$ has great influence on its thermal performance. It is a measure of how fast heat will flow in that material. In heat transfer analysis, a material is normally assumed to be isotropic. It has uniform properties in all directions. Therefore, it is common practice to evaluate the thermal conductivity $k$ at the average temperature and treat it as a constant in calculations [1]. This assumption is realistic for most materials, but some materials, such as laminated composite materials and wood, they are anisotropic and exhibit different structural characteristics in different directions. For example, the thermal conductivity of wood across the grain is different than that parallel to the grain. In such cases the thermal conductivity $k$ should be expressed as a tensor quantity to account for the variation with direction. Shiah and Tan [2-3] developed an exact transformation approach that can be applied to numerically treat the two-dimensional and even three-dimensional anisotropic problems in field theory by the boundary element method (BEM). The thermal conductive theory of anisotropic materials plays an important role in many areas of science and technology. But works about it are still limited and relatively few reported.

Double skin envelope or double skin facade (DSF) is widely applied in building and architecture area. Experimental and analytical studies have been carried out on the thermal characteristics of DSF [4-6]. Pasquay [7] presented the advantages and disadvantages of DSF on the basis of actual measurements of high-rise buildings. Ding et al. [8] finished

*Address correspondence to this author at the College of Urban Construction, Wuhan University of Science and Technology, No. 15 mail box, No. 199 Xiongchu Avenue, Wuhan 430070, China; Tel: +86-027-68893562; Fax:+86-027-68893641; E-mail: shunyusu@163.com reduced-scale model experiments and a computational fluid dynamics analysis to evaluate the natural ventilation performance in the prototype of an eight-storey building with DSF in the south. Wong et al. [9] reported that DSF used on the eastern and western walls of building can effectively conserve cooling energy by shielding buildings from solar radiation and reducing the heat by natural ventilation. Nowadays studies about DSF are undertaken in different climatic conditions such as hot and humid; moderate; cold and hot arid climates [10-13]. Moreover, detailed information on the thermal distribution in DSF is necessary for building envelope so as to not only provide comfortable indoor environment but also save energy.

The purpose of this paper is to analyze the thermal conductive characteristics of anisotropic materials, and to discuss the application of anisotropic material of thermal conductivity in double skin envelope system so as to make use of its advantage and avoid its disadvantage.

\section{CONDUCTIVE MECHANISM OF ANISOTROPIC MATERIALS OF THERMAL CONDUCTIVITY}

Comparing with isotropic material, the conductive process of anisotropic material has two important differences. One is that the thermal conductivity of anisotropic material is different in different directions. The other is that the magnitude of heat transfer rate of anisotropic material in one direction is not only relevant to the temperature gradient in this direction, but also relevant to the temperature gradient which is normal to the direction [14]. In rectangular coordinates $\left(x_{1}, x_{2}\right)$ for two-dimensional conduction, the magnitudes of heat flux $q_{\mathrm{x} 1}$ and $q_{\mathrm{x} 2}$ in $x_{1}$ and $x_{2}$ directions can be determined from Fourier's law as 


$$
\begin{aligned}
& q_{x_{1}}=-k_{11} \frac{\partial t}{\partial x_{1}}-k_{12} \frac{\partial t}{\partial x_{2}} \\
& q_{x_{2}}=-k_{21} \frac{\partial t}{\partial x_{1}}-k_{22} \frac{\partial t}{\partial x_{2}}
\end{aligned}
$$

where $t$ represents the temperature in the anisotropic material, and $k_{\mathrm{ij}},(i, j=1,2)$ are the thermal conductivities, where from thermodynamic principles and Onsagar's reciprocity relation, the coefficients must satisfy the following relations:

$k_{11}>0, k_{22}>0, k_{11} k_{22}-k_{12}{ }^{2}>0, k_{11}=k_{12}$

To solve the anisotropic problem is evidently a more complicated process since $k_{\mathrm{ij}}$ are invariant coefficients with respect to $x_{1}$ - and $x_{2}$-axes in rectangular coordinate system. A commonly adopted approach to treat the anisotropic problem is to determine the principal axes $\left(\xi_{1}, \xi_{2}\right)$ by rotating the original rectangular axes so that the cross-derivative terms will disappear. In other words, in rectangular coordinates $\left(\xi_{1}\right.$, $\xi_{2}$ ) for two-dimensional conduction, the magnitude of heat flux in the $\xi_{\mathrm{i}}(i=1,2)$ direction is relevant to the temperature gradient in this direction, but is not relevant to the temperature gradient in orthogonal axis. The magnitudes of heat flux can also be expressed from Fourier's law as

$$
\begin{aligned}
& q_{\xi_{1}}=-k_{1} \frac{\partial t}{\partial \xi_{1}} \\
& q_{\xi_{2}}=-k_{2} \frac{\partial t}{\partial \xi_{2}}
\end{aligned}
$$

where $k_{1}$ and $k_{2}$ represent the principal thermal conductivities.

Fig. (1) shows the two-dimensional steady-state conduction of an anisotropic material. There is a laminated composite plate with anisotropic conductivity. The inclined lines in the figure express laminated surfaces. The directions along the laminated surface and its normal are thermal conductivity principal axes $\xi_{1}$ and $\xi_{2}$. The thermal conductivities along the principal axes $\xi_{1}$ and $\xi_{2}$ are $k_{1}$ and $k_{2}$. The $x_{1}$ axis represents the direction of isothermal surface. Rectangular coordinates $\left(x_{1}, x_{2}\right)$ are inconsistent with the principal axes $\xi_{1}$ and $\xi_{2}$. $\beta$ represents the anisotropic angle in the figure.

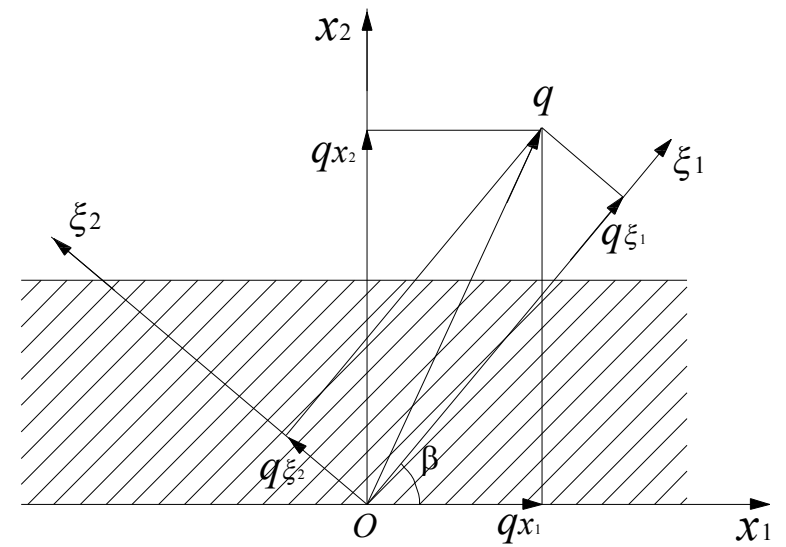

Fig. (1). Two-dimensional steady-state conduction of the anisotropic material.
Since temperature gradient and heat flux are not relevant to coordinate system in the same object, the following may be obtained:

$$
\begin{aligned}
q_{x_{1}} & =q_{\xi_{1}} \cos \beta-q_{\xi_{2}} \sin \beta \\
q_{x_{2}} & =q_{\xi_{1}} \sin \beta+q_{\xi_{2}} \cos \beta \\
\frac{\partial t}{\partial x_{1}} & =\frac{\partial t}{\partial \xi_{1}} \cos \beta-\frac{\partial t}{\partial \xi_{2}} \sin \beta \\
\frac{\partial t}{\partial x_{2}} & =\frac{\partial t}{\partial \xi_{1}} \sin \beta+\frac{\partial t}{\partial \xi_{2}} \cos \beta
\end{aligned}
$$

From Eq. (6) to Eq. (9), the following may be deduced:

$$
\begin{aligned}
& q_{x_{1}}=-\left(k_{1} \cos ^{2} \beta+k_{2} \sin ^{2} \beta\right) \frac{\partial t}{\partial x_{1}}-\left(k_{1}-k_{2}\right) \sin \beta \cos \beta \frac{\partial t}{\partial x_{2}} \\
& q_{x_{2}}=-\left(k_{1}-k_{2}\right) \sin \beta \cos \beta \frac{\partial t}{\partial x_{1}}-\left(k_{1} \sin ^{2} \beta+k_{2} \cos ^{2} \beta\right) \frac{\partial t}{\partial x_{2}}
\end{aligned}
$$

If the two surfaces of the laminated composite plate maintain constant temperatures of $t_{\mathrm{w} 1}$ and $t_{\mathrm{w} 2}$. There will be one- dimensional temperature field in the plate, and

$$
\begin{aligned}
& \frac{\partial t}{\partial x_{1}}=0 \\
& \frac{\partial t}{\partial x_{2}}=-\frac{t_{w 1}-t_{w 2}}{\delta}
\end{aligned}
$$

where $\delta$ represents thickness of the laminated composite plate.

So that

$$
\begin{aligned}
& q_{x_{1}}=-\left(k_{1}-k_{2}\right) \sin \beta \cos \beta \frac{\partial t}{\partial x_{2}} \\
& =\left(k_{1}-k_{2}\right) \sin \beta \cos \beta \frac{t_{w 1}-t_{w 2}}{\delta} \\
& q_{x_{2}}=-\left(k_{1} \sin ^{2} \beta+k_{2} \cos ^{2} \beta\right) \frac{\partial t}{\partial x_{2}} \\
& =\left(k_{1} \sin ^{2} \beta+k_{2} \cos ^{2} \beta\right) \frac{t_{w 1}-t_{w 2}}{\delta}
\end{aligned}
$$

Equations (14) and (15) indicate, for anisotropic material, the temperature gradient in one direction develops magnitude of heat transfer rate in orthogonal direction.

\section{ADVANTAGE OF ANISOTROPIC MATERIALS OF THERMAL CONDUCTIVITY}

Basing on above analysis, it can be concluded that the heat flux vector is commonly not perpendicular to the isothermal surface in anisotropic material. For thermal conduction in a plate as Fig. (1) shows, the plate conductive heat flux $q_{x 2}$ is more concerned. The apparent thermal conductivity is defined 


$$
k^{*}=k_{1} \sin ^{2} \beta+k_{2} \cos ^{2} \beta
$$

Therefore,

$q_{x_{2}}=-k^{*} \frac{t_{w 2}-t_{w 1}}{\delta}$

For thermal conduction in the plate made of isotropic material, the conductive heat flux across it is expressed as

$q_{x_{2}}^{\prime}=-k_{1} \frac{t_{w 2}-t_{w 1}}{\delta}$

If $k_{1}=k_{2}$, then the apparent thermal conductivity $k^{*}=k_{1}$. The thermal conductive material becomes isotropic. Ordinarily, $0<k_{2}<k_{1}$. Table 1 shows the apparent thermal conductivity $k^{*}$ varies with the anisotropic angle $\beta$. For special case, while $k_{2}=0, k^{*}=k_{1} \sin ^{2} \beta<k_{1}$; while $\beta=0^{\circ}, k^{*}=k_{2}$. Since the value of $k_{2}$ is more lower than that of $k_{1}$, the thermal conductive material becomes a poor heat conductor or insulator; while $\beta=90^{\circ}, k^{*}=k_{1}$. The thermal conductive performance is very good for this material, or this material is a good heat conductor, but the insulating effect is consequentially worse for it.

The results of Table $\mathbf{1}$ show that generally the apparent thermal conductivity $k^{*}<k_{1}$. There fore, $q_{x_{2}}<q_{x_{2}}^{\prime}$. In other words, if the thermal conductivity of isotropic material in thickness direction is equal to that of anisotropic material in laminated direction, the magnitude of heat flux across the plate made of anisotropic material is less than that made of isotropic material.

This advantage of anisotropic material can be made use in building envelope. Fig. (2) shows the conduction of building envelope made of anisotropic material in summer while the outdoor temperature is higher than that of indoor. The heat transfer rate vector $q$ is not perpendicular to the building wall. It will develop partial magnitude paralleling to the wall. Then the real heat flux across the building envelope from outdoor to indoor decreases when the wall temperatures of two sides maintain constant respectively. If $0<k_{2}<k_{1}$, it can easily come true by laying out laminated composite material in reasonable direction, the partial magnitude of heat transfer rate will flow upwards. Finally, it will spread to the ambient on the roof of the single-storey building by thermal convection with the around air. Therefore, the indoor cooling load in summer decreases while the anisotropic material is used as building envelope.

Fig. (3) shows the conduction of building envelope made of anisotropic material in winter while the indoor tempera- ture is higher than outdoor. The real heat flux across the building envelope from indoor to outdoor decreases when the wall temperatures of two sides maintain constant respectively. The heat transfer rate vector $q$ will develop partial magnitude paralleling to the wall. If $0<k_{2}<k_{1}$, the partial magnitude of heat transfer rate will flow downwards. It can partially spread to the indoor air by thermal convection. Then, the indoor heating load in winter may decrease a little while the anisotropic material is used as building envelope.

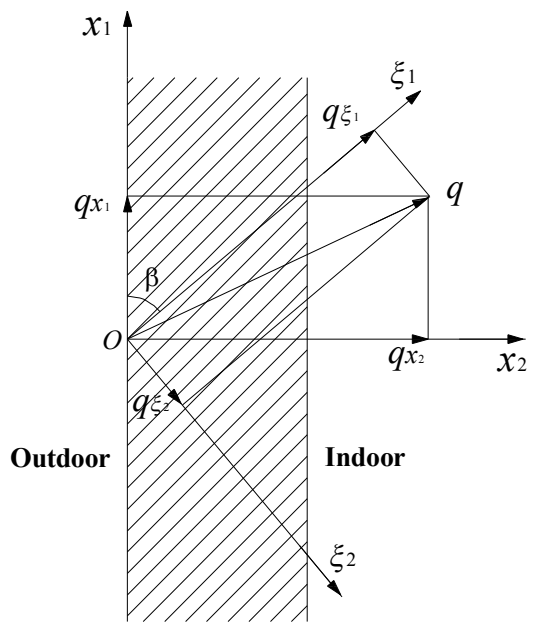

Fig. (2). Conduction of the anisotropic material in summer.

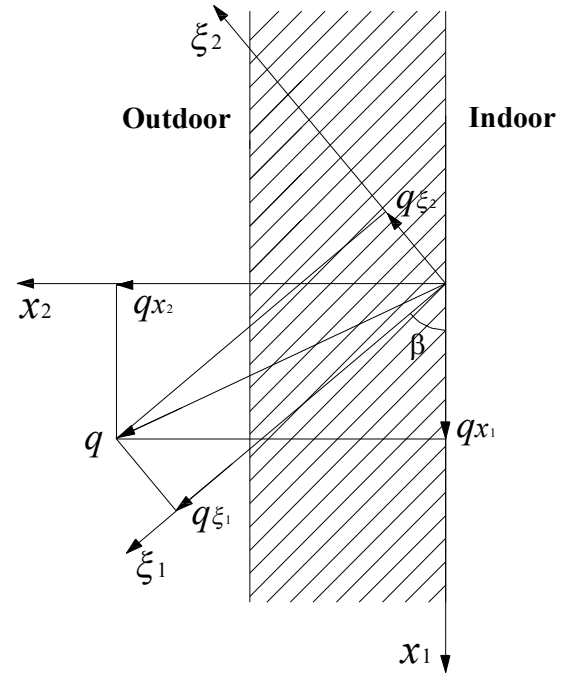

Fig. (3). Conduction of the anisotropic material in winter.

\section{DOUBLE SKIN ENVELOPE SYSTEM AND LAMI- NATED COMPOSITE ANISOTROPIC MATERIALS}

Although the anisotropic material of thermal conductivity made of laminated composite has its advantage used in

Table 1. The Apparent Thermal Conductivities Varying with the Anisotropic Angles

\begin{tabular}{|c|c|c|c|c|c|}
\hline $\boldsymbol{\beta}$ & $\mathbf{0}^{\circ}$ & $\mathbf{3 0}^{\circ}$ & $\mathbf{4 5}^{\circ}$ & $\mathbf{6 0}^{\circ}$ & $\mathbf{9 0}^{\circ}$ \\
\hline \hline$k^{*}$ & $k_{2}$ & $\frac{k_{1}+3 k_{2}}{4}$ & $\frac{k_{1}+k_{2}}{2}$ & $\frac{3 k_{1}+k_{2}}{4}$ & $k_{1}$ \\
\hline
\end{tabular}


building envelope, it is still imperfect when it used in multistorey building or high-rise building. Since partial magnitude of heat transfer rate will flow upwards in laminated composite material in summer, for single-storey building, this part of heat will spread to the ambient on the roof of the building by thermal convection with the around air; but for multi-storey building or high-rise building, it may spread to the indoor of upper storey by thermal convection with the indoor air when it flows upwards in the building envelop. In order to avoid this shortcoming, it could be related to double skin facade in building.

The technique of double skin facade has been successfully applied in many building designs. Double skin facade using as the building envelope consists of an outer glazed skin and an inner glazed or partially glazed one. The two skins together with top and bottom dividing plates form a cavity. This air layer works as a thermal buffer zone. And this kind of building envelope is proposed not only to reduce noise from outdoors, but most importantly, to decrease indoor heating load in winter by increasing outside temperature of the inner facade, using solar incidence, and decrease indoor cooling load in summer by lowering the temperature via ventilation in the cavity.

Fig. (4) shows schematic diagram of this double skin envelope in summer. The top and bottom dividing plates are open in summer, and outdoor air is induced into the cavity and naturally ventilated through it by buoyancy arising from the outside and inside temperature difference of the cavity. Heat in the cavity and from the two skins spread out in this convection process. Fig. (5) shows schematic diagram of this

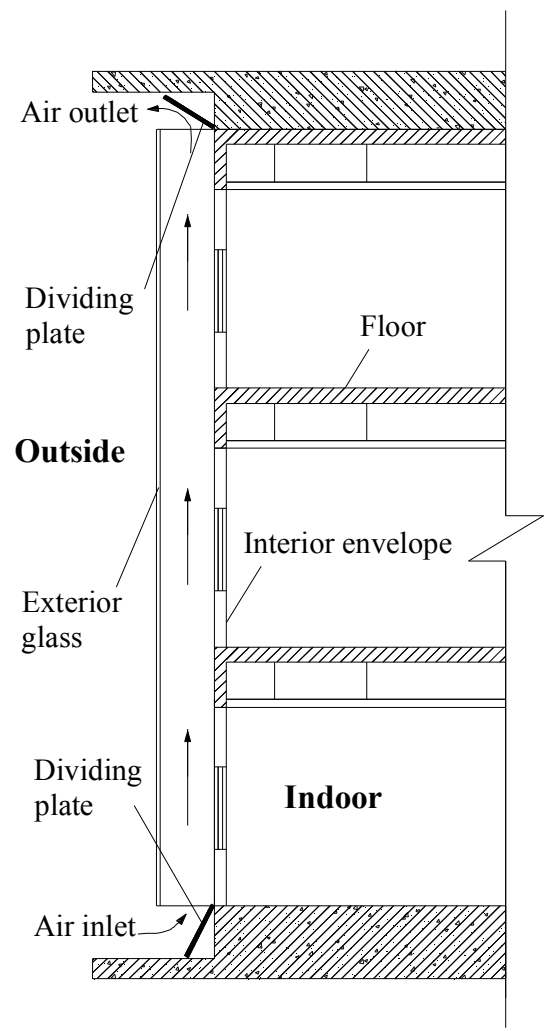

Fig. (4). Schematic diagram of double skin envelope in summer. double skin envelope in winter. The top and bottom dividing plates are close. Heat from sunshine stores in the cavity and then transfers to indoor through the interior envelope. This building envelope system is considered to be an environment-friendly design since it utilizes natural ventilation and sunshine so that comfort can be achieved with less energy consumption [15]. Double skin envelope used in summer could avoid the disadvantage of anisotropic material of thermal conductivity by ventilation in its cavity. Ventilation in the cavity of this double skin building envelope system can be natural, mechanical or a combination of both [16].

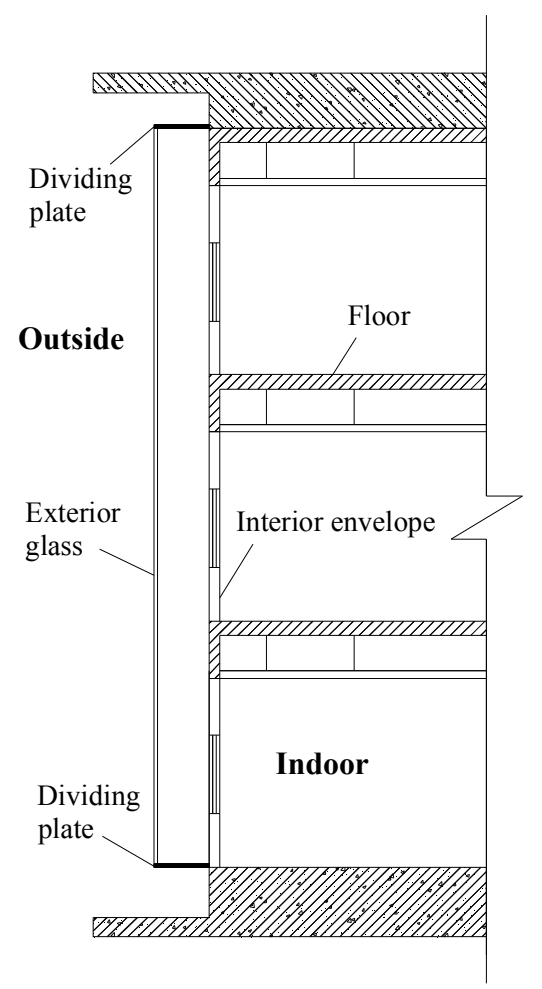

Fig. (5). Schematic diagram of double skin envelope in winter.

\section{APPLICATION OF LAMINATED COMPOSITE WITH ANISOTROPIC THERMAL CONDUCTIVITY IN DOUBLE SKIN ENVELOPE}

The selection of building material has important effect to the design of building thermal performance and energy saving. According to above analysis, laminated composite with anisotropic thermal conductivity could be applied in double skin envelope. As Fig. (2) shows, the partial magnitude of heat transfer rate in the building envelope with anisotropic material will flow upwards in summer. It will spread to outside on the roof of the single-storey building by thermal convection with the around air. But in multi-storey or high-rise building, this partial magnitude of heat transfer rate will flow upstairs to the interior envelope of higher floor. It may transfer to indoor of higher floor by convection between the interior envelope surface and indoor air of the higher floor. Thus the indoor cooling load of the higher floor will increase.

To avoid this disadvantage, the laminated composite with anisotropic thermal conductivity could be used as the mate- 
rial of interior envelope in double skin envelope. The interior envelope may be made of laminated glaze or other laminated material as Fig. (2) or Fig. (3) shows. The top and bottom dividing plates of the double skin envelope will be open in summer. The heat in the surface of interior envelope will be brought out by flowing air through the cavity. And in winter, the dividing plates will be close. In this case, the heat in the surface of interior envelope can only transfer to the air in the close cavity. But this heat transfer is limited. In fact, the cavity is warmer than outside. And with solar incidence on the facade of exterior envelope, its temperature increases. Comparing with outside temperature in winter, the higher cavity temperature has a significant role in reducing heat loss from the building. It can be concluded that this combined double skin envelope system, while the interior envelope is made of laminated composite with anisotropic thermal conductivity, will lower indoor cooling load in summer as well as indoor heating load in winter. The effect of energy saving is obvious in summer since the partial magnitude of conductive heat in the interior envelope could be brought out from the cavity by natural ventilation through it.

\section{CONCLUSIONS}

The thermal performance of conductivity anisotropic material was analyzed in this paper. The characteristics in anisotropic materials are different from those in isotropic materials. The heat flux vector is commonly not perpendicular to the isothermal surface in anisotropic material. The magnitude of heat transfer rate of anisotropic material in one direction is not only relevant to the temperature gradient in this direction, but also relevant to the temperature gradient which is normal to the direction.

The double skin facade in winter preheats the interior space of the envelope and decreases the heating load. And in summer, the ventilation of the cavity can effectively lower the cooling load. The interior envelope in double skin system may be made of laminated glaze or other laminated material with anisotropy. Basing on the combination of anisotropic material and double skin envelope, the indoor cooling and heating load decrease in summer and winter respectively while the anisotropic material was used as interior building envelope.

\section{CONFLICTS OF INTEREST}

None Declared

\section{ACKNOWLEDGMENTS}

The authors acknowledge the financial support from Doctoral Science Research Foundation of Wuhan University of Science and Technology. The authors also would like to thank the Organizing Committee of the 2nd International Conference on Manufacturing Science and Engineering (ICMSE 2011). The paper was revised according to their comments.

\section{REFERENCES}

[1] Y. A. Cengel, Heat transfer: a practical approach, $2^{\text {nd }}$ ed., New York: McGraw Hill, 2003.

[2] Y. C. Shiah and C. L. Tan, "BEM treatment of two-dimensional anisotropic field problems by direct domain mapping", Engng. Anal. Bounda. Elem., vol. 20, pp. 347-351, 1997.

[3] Y. C. Shiah and C. L. Tan, "BEM treatment of three-dimensional anisotropic field problems by direct domain mapping", Engng. Anal. Bounda. Elem., vol. 28, pp. 43-52, 2004.

[4] H. Manz, "Total solar energy transmittance of glass double facades with free convection", Energy Build., vol. 36, pp. 127-136, 2004.

[5] E. Gratia, A. D. Herde, "The most efficient position of shading devices in a double-skin facade", Energy Build., vol. 39, pp. 364-373, 2007.

[6] T. Hiroaki, O. Masaya, T. Hideki, Y. Y. Gyu, W. Kenji, "Thermal characteristics of a double-glazed external wall system with roll screen in cooling season", Build. Environ., vol. 44, pp. 1509-1516, 2009.

[7] T. Pasquay, "Natural ventilation in high-rise buildings with double facades, saving or waste of energy", Energy Build., vol. 36, pp. 381389, 2004.

[8] W. T. Ding, Y. Hasemi, T. Yamada, "Natural ventilation performance of a double-skin facade with a solar chimney", Energy Build., vol. 37, pp. 411-418, 2005.

[9] N. H. Wong, L. Wang, N. C. Aida, R. P. Anupama, X. Wei, "Effects of double glazed facade on energy consumption, thermal comfort and condensation for a typical office building in Singapore", Energy Build., vol. 37, pp. 563-572, 2005.

[10] P. C. Wong, D. Parsad, M. Behnia, "A new type of double skin facade configuration for the hot and humid climate", Energy Build., vol. 40, pp. 1941-1945, 2008.

[11] E. Gratia, A. D. Herde, "Optimal operation of a south double-skin facade", Energy Build., vol. 36, pp. 41-60, 2004.

[12] R. Hossegen, B. J. Wachenfeldt, S. O. Hanssen, "Building simulation as an assisting tool in decision making, case study: with or without a double-skin facade?", Energy Build., vol. 40, pp. 821-827, 2008.

[13] N. Hashemi, R. Fayaz, M. Sarshar, "Thermal behaviour of a ventilated double skin facade in hot arid climate", Energy Build., vol. 42, pp. 1823-1832, 2010.

[14] Q. S. Yang and B. Y. Pu, Advanced heat transfer, 2nd ed., Shanghai: Shanghai Jiao Tong University Press, 2001. (In Chinese)

[15] Y. M. Kim, S. Y. Kim, S. W. Shin and J. Y. Sohn, "Contribution of natural ventilation in a double skin envelope to heating load reduction in winter", Build. Environ., vol. 44, pp. 2236-2244, 2009.

[16] D. Saelens, S. Roels, H. Hens, "Strategies to improve the energy performance of multiple-skin facades", Build. Environ., vol. 43, pp. $638-650,2008$ 